\title{
GMR
}

\section{Expression characteristics of MMP-2 and MMP-9 in guinea pig ovaries during the estrous cycle}

\author{
J.R. Li and T. Shen \\ College of Agriculture and Bioengineering, Jinhua Polytechnic, Jinhua, China \\ Corresponding author: J.R. Li \\ E-mail: lijornsky@163.com
}

Genet. Mol. Res. 14 (4): 17329-17340 (2015)

Received June 29, 2015

Accepted September 2, 2015

Published December 21, 2015

DOI http://dx.doi.org/10.4238/2015.December.21.1

\begin{abstract}
We investigated the expression characteristics of matrix metalloproteinases (MMP)-2 and MMP-9 in the ovaries of normal adult guinea pigs during the estrous cycle. The estrous cycle was classified according to the appearance of the vaginal orifice and by vaginal exfoliated cell smears. Guinea pigs that had experienced at least two normal estrous cycles were selected. Ovaries were collected from guinea pigs at proestrus or metestrus $(\mathrm{N}=6)$. The expression characteristics of steroidogenic acute regulatory protein (StAR), MMP-2, and MMP-9 were investigated by hematoxylin and eosin staining and immunohistochemical staining. The results showed that MMP-2, MMP-9, and StAR were significantly expressed in the granulosa and thecal cells of the ovarian atretic follicles during proestrus, and were strongly expressed in the corpus luteum during metestrus.
\end{abstract}

Key words: Guinea pigs; Ovary; MMP-2; MMP-9; StAR 


\section{INTRODUCTION}

Matrix metalloproteinases (MMPs) are endogenous Zn-containing proteases with a catalytic function domain and a polypeptide domain (Massova et al., 1998; Rivera et al., 2010). MMPs are responsible for the remodeling of the extracellular matrix (ECM) (Raza and Cornelius, 2000; Di Nezza et al., 2002; Kessenbrock et al., 2010). MMPs are divided into soluble and membranous MMPs. The soluble MMPs include collagenases (MMP-1, -8, -13, and -18), gelatinases (MMP-2 and -9), matrilysins (MMP-3, -10, and -11), matrix enzymes (MMP-7 and -26), and other enzymes (MMP-12, -19, -20, -21, -23, -27, and -28). Membranous MMPs include MMP-14, -15, -16, -17, -24, and -25 (Nagase and Woessner, 1999; Lohi et al., 2001; Nagase et al., 2006). MMPs play important roles in the development of many organisms; for example, they are involved in organ morphogenesis, ovulation, apoptosis, angiogenesis, and embryogenesis (Shi et al., 1998; Vu and Werb, 2000; Roy et al., 2006; Jones, 2014). MMPs are involved in regulating the ability of extracellular molecules and proteins to divide (Sternlicht et al., 2000), and in the processing of signaling molecules (Roy et al., 2009). MMPs are inhibited by tissue inhibitors of MMPs (TIMPs). TIMPs connect the regulating activity through non-covalent bonds and inhibit the functions of MMPs through zinc reactions (Brew et al., 2000).

Through ECM remodeling, MMPs cooperate with TIMPs at the ovulatory period to play important roles in ovary and follicular development (Bagavandoss, 1998; Cooke et al., 1999; Baker et al., 2000; McCaffery et al., 2000). MMP-2 and MMP-9 (gelatinases A and B) contain a fibronectinlike functional domain in their catalytic functional domains (Bode et al., 1999) and are involved in the degradation of polysaccharide core proteins, such as collagens (IV, V, and XI), laminins, and cartilagins (Nagase et al., 2006). Gelatinases are indispensable in matrix membrane remodeling (Stetler-Stevenson, 1994). MMP-2 and MMP-9 have been identified at the follicular theca and the matrix of developing follicles in rodents (Garcíaet al., 1997). As reported, intra-follicular levels of MMP-2 and MMP-9 increase during follicular atresia in normal sheep (Huet et al., 1998). A very high follicular atresia rate is usually accompanied by high levels of MMP-2 and MMP-9 (Shalev et al., 2001; Ben-Shlomo et al., 2003). However, it is not clear whether MMPs and TIMPs are involved in the different stages of the estrous cycle.

Guinea pigs are very popular experimental animals because they are easier to operate on than larger animals, have an estrous cycle that is more like the human menstrual cycle than the estrous cycle in other rodents, and have obvious estrous characteristics. The ovary follicular period of guinea pigs is very similar to that of humans (van Kan et al., 2009), and they are therefore very good animal models for research on human propagation (Kulduk et al., 2014; Sun et al., 2014).

In this study, MMP-2 and MMP-9 were used as indicators of ECM remodeling variation to investigate whether ovary gelatinases (MMP-2 and MMP-9) are involved at proestrus and metestrus in guinea pigs. Moreover, the immunolocalization of steroidogenic acute regulatory protein (StAR) in the ovaries of guinea pigs was detected, because StAR is an important indicator of steroid synthesis (Clark et al., 1997).

\section{MATERIAL AND METHODS}

\section{Animals and experimental design}

White female adult guinea pigs (Caviaporcellus, 400-600 g) were purchased from the 
Jinhua Laboratory Animal Center. The estrous cycles of the guinea pigs were continuously observed. The appearance of the vaginal orifice was observed and exfoliated cell smears were prepared. Guinea pigs that had experienced two continuous estrous cycles (16-18 days) were selected as the test animals. Guinea pigs at proestrus or metestrus were selected $(N=6)$. The guinea pigs were sacrificed under anesthesia and their ovaries were rapidly separated and fixed in $4 \%$ paraformaldehyde for $24 \mathrm{~h}$. The ovaries were then cut with paraffin into tissue slices $(5 \mu \mathrm{m})$ and stained with hematoxylin and eosin. With StAR as the indicator of ovary steroid synthesis, and MMP-2 and MMP-9 as the indicators of ovarian tissue remodeling, the slices were stained immunohistochemically. The expression characteristics of follicular gelatinases during ovary development were then investigated.

\section{Reagents}

The reagents comprised MMP-2 (SC-8835, lot: L5281); MMP-9 (SC-6840, lot: L8393); StAR (SC-25806, lot: 12308); sheep IgG-horseradish peroxidase (HRP) (SC-2354, lot: H2213) (Santa Cruz Biotechnology Inc., TX, USA); a rabbit IgG immunohistochemical (IHC) kit (SA2002, lot: 10C09A) (Wuhan Boster Bioengineering Co., Ltd., Wuhan, China); 3,3'-diaminobenzidine (JD091) (Gen-View Scientific Inc., FL, USA); and Wright-Giemsa stain solution (BA-4017) (Zhuhai Beisuo Biological Technology Co., Ltd., Zhuhai, China). All other reagents were analytically pure and were made in China.

\section{Vaginal exfoliated cell smears}

The shapes of the vaginal orifices were observed first. Then, medical cotton swabs soaked in normal saline were gently inserted into the vaginas. After rotation, the cotton swabs were immediately taken out and the mucilage was evenly coated onto slides, which were rapidly fixed in a flame to avoid cell deformation. After fixing, $0.5 \mathrm{~mL}$ Wright-Giemsa A solution was dripped onto the slides. After the A solution had covered the entire surface of each slide for $1 \mathrm{~min}$, WrightGiemsa B solution ( $1 \mathrm{~mL}$ ) was dripped onto the A solution. A rubber pipette bulb was used to gently blow solutions $A$ and $B$ for full mixing for $5 \mathrm{~min}$. After staining, water-washing, and drying, the slices were observed under a microscope.

\section{IHC staining}

After dewaxing, the slides were placed in a sodium citrate/phosphate-buffered saline solution (PBS; $200 \mathrm{~mL}$ ) and heated in a microwave oven (P70D20TL-D4, Galanz) at $100^{\circ} \mathrm{C}$ for $4 \mathrm{~min}$. The door of the microwave oven was then opened and the slides were heated for a further $3 \mathrm{~min}$. After heat repair, bovine serum albumin (BSA) was used to seal the slides. After sealing, the BSA-PBS solution on the slides was washed off and replaced with a primary antibody to cover the ovarian tissues (PBS was used in the control group). After incubation in an incubation box with the primary antibodies for $12 \mathrm{~h}\left(4^{\circ} \mathrm{C}\right)$, the secondary antibodies were applied for incubation at room temperature for $2 \mathrm{~h}$. After that, the third antibodies were applied for incubation at room temperature for $1 \mathrm{~h}$ (for MMP-2 and MMP-9, IgG-HRP as secondary antibodies were used, without the third antibodies). After incubation, the slides were put into a 3,3'-diaminobenzidine solution for staining for $10 \mathrm{~s}$. After washing with water for $5 \mathrm{~min}$, the slides were then stained with hematoxylin for $10 \mathrm{~s}$, then washed with water for $5 \mathrm{~min}$. The sections were finally washed, sealed, and observed under the microscope. 


\section{Image analysis}

The ovary slides were observed under a microscope (BX51, Olympus). Ovary virtual images were then obtained using an optical imaging system (XC10, Olympus). Finally, the images were analyzed using OlyVIA 2.4 (Olympus Soft Imaging Solutions, GmbH, Japan).

\section{RESULTS}

\section{Determination of estrous cycle in guinea pigs}

Continuous vaginal orifice observation showed slight vaginal opening (Figure $1 \mathrm{~A}$ ), or a small amount of secretion, and a red vaginal orifice at proestrus. Slight vaginal opening (Figure 1B), secretion, and a red vulva were observed during estrous. Slight vaginal closing (Figure 1C), or solid secretions, and a light-colored vaginal orifice were observed at metestrus. Slight vaginal closing (Figure 1D) and no secretion were observed at diestrus, while the red and swollen vaginal orifices disappeared or became white.
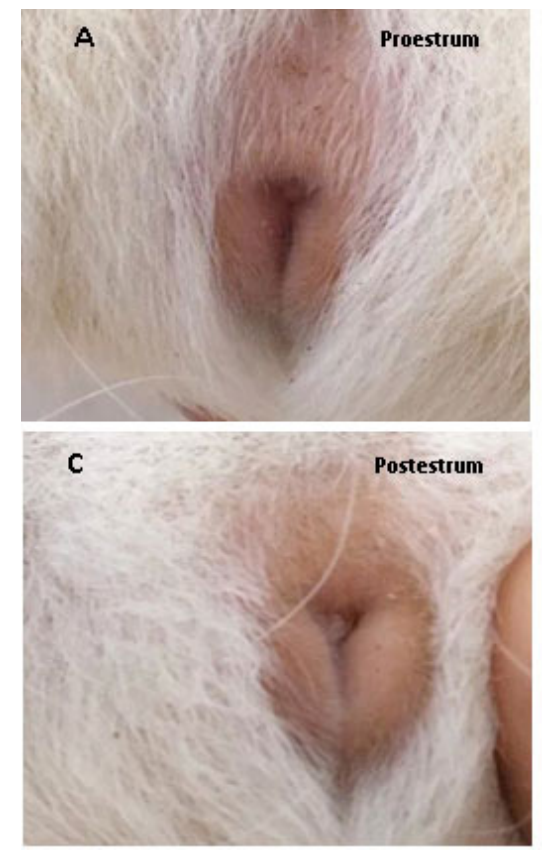
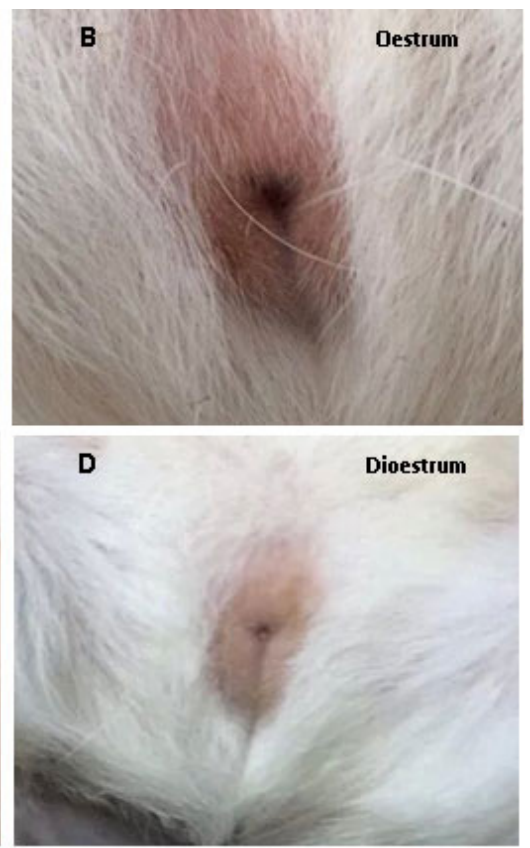

Figure 1. Vaginal orifice appearance at different estrous stages.

The vaginal exfoliated cells at the four estrous stages were markedly different. The cell smears at proestrus contained a small number of fully cornified epithelial cells (ECs, Figure 2A). The smears at estrous contained a large number of fully cornified ECs (Figure 2B). The smears at metestrus contain abundant white blood cells (WBCs) and a small number of ECs (Figure 2C). The smears at diestrus contained a small number of WBCs (Figure 2D). 

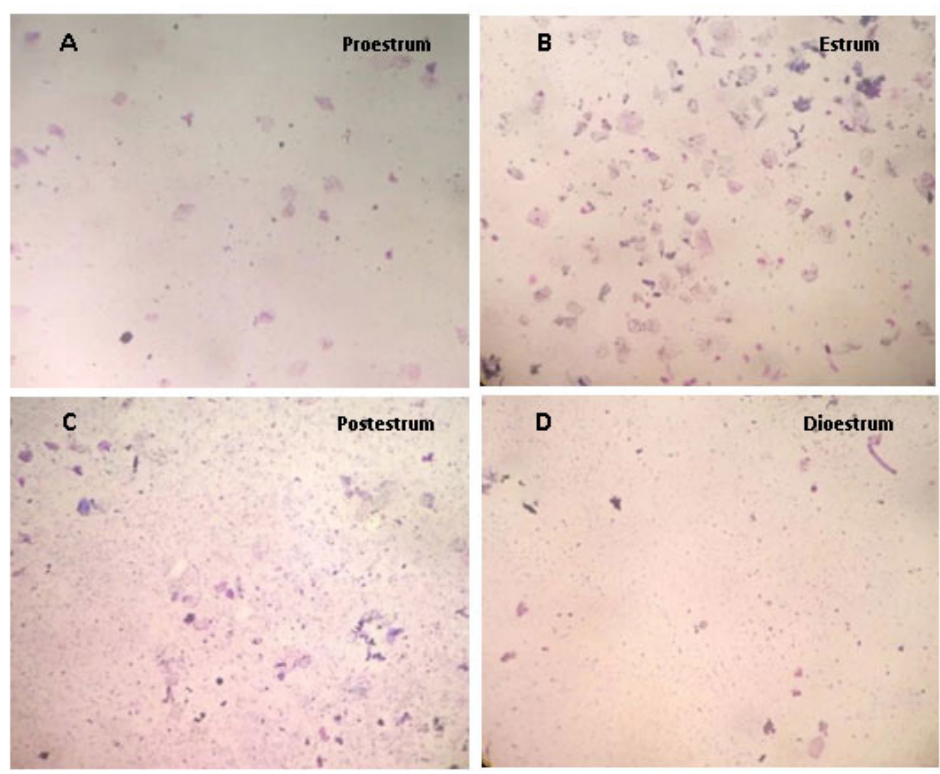

Figure 2. Vaginal exfoliated cell smears at different estrous stages.

\section{Ovary shapes at proestrus and metestrus}

The ovaries at proestrus showed very large antral follicles (Figure $3 \mathrm{~A}$ ) and atretic follicles (Figure 3A1), accompanied by a small number of secondary follicles (Figure 3B). The secondary follicles contained small follicular cavities. The oocytes were healthy and the granulosa cells and thecal cells were closely connected (Figure 3B1). Some of the large-volume follicles were atretic to different degrees (Figure $3 \mathrm{C}$ ), and a large number of granulosa cells fell down into the follicular cavity (Figure 3C1).
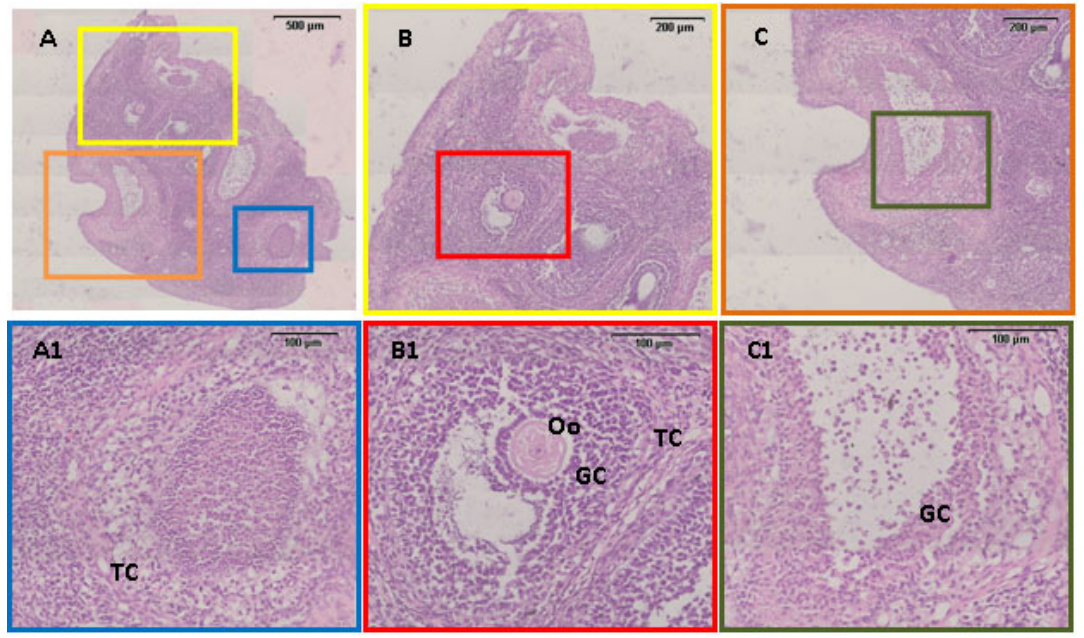

Figure 3. Proestrus ovary shapes. $\mathrm{Oo}=$ oocyte; $\mathrm{GC}=$ granulosa cells; $\mathrm{TC}=$ theca cells. 
The metestrus ovaries showed corpus luteum after ovulation (Figure 4A), while the large follicular cavities contained a small number of granulosa cells (Figure 4A1), or the granulosa cell layer and the thecal cell layer were evidently separated (Figure 4A2). The follicular walls showed abundant original follicles (Figure 4B, B1), accompanied by a small number of primary follicles (Figure 4B2). The ovaries also showed secondary follicles (Figure 4C, C2) and late-atresia follicles (Figure 4C1). The follicular cavities did not show oocytes, but only a deformed transparent zone.
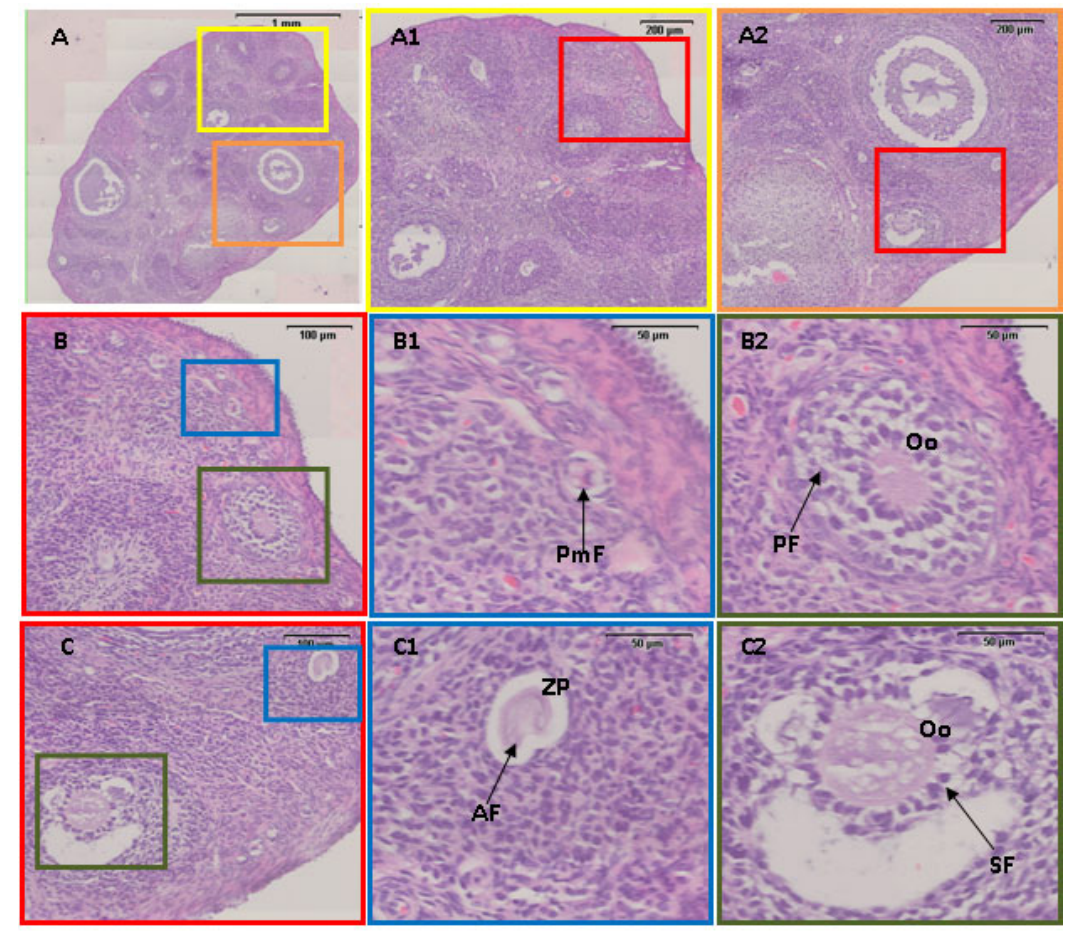

Figure 4. Ovary shapes at metestrus. Oo = oocyte; $\mathrm{ZP}=$ zona pellucida; $\mathrm{AF}=$ atretic follicle; $\mathrm{PmF}=$ primordial follicle; $\mathrm{PF}=$ primary follicle; $\mathrm{SF}=$ secondary follicle.

\section{Ovary StAR expression levels at proestrus and metestrus}

In the proestrus ovaries, the StAR expression levels in the atretic follicles were evident compared with the negative control (Figure 5A, A1, and A2). StAR was only expressed in the atretic antral follicles and atretic non-antral follicles (Figure 5B), but not in the developing follicles. In the atretic antral follicles, StAR was only expressed in the basal membrane cell layer, but not markedly in the granulosa cell layer (Figure 5B1). StAR was markedly expressed in the basal membranedifferentiated cells of the non-antral atretic follicles (Figure 5B2).

In the metestrus ovaries, compared with the negative control (Figure 6A, A1, and A2), StAR was only markedly expressed in the corpus luteum (Figure 6B), but not in the antral follicles. Compared with the control group, StAR was strongly expressed in the luteal cells and weakly expressed in the follicular theca cell layer (Figure 6A1,6B1), but the expression levels in the luteal cells were the same (Figure 6B2). 

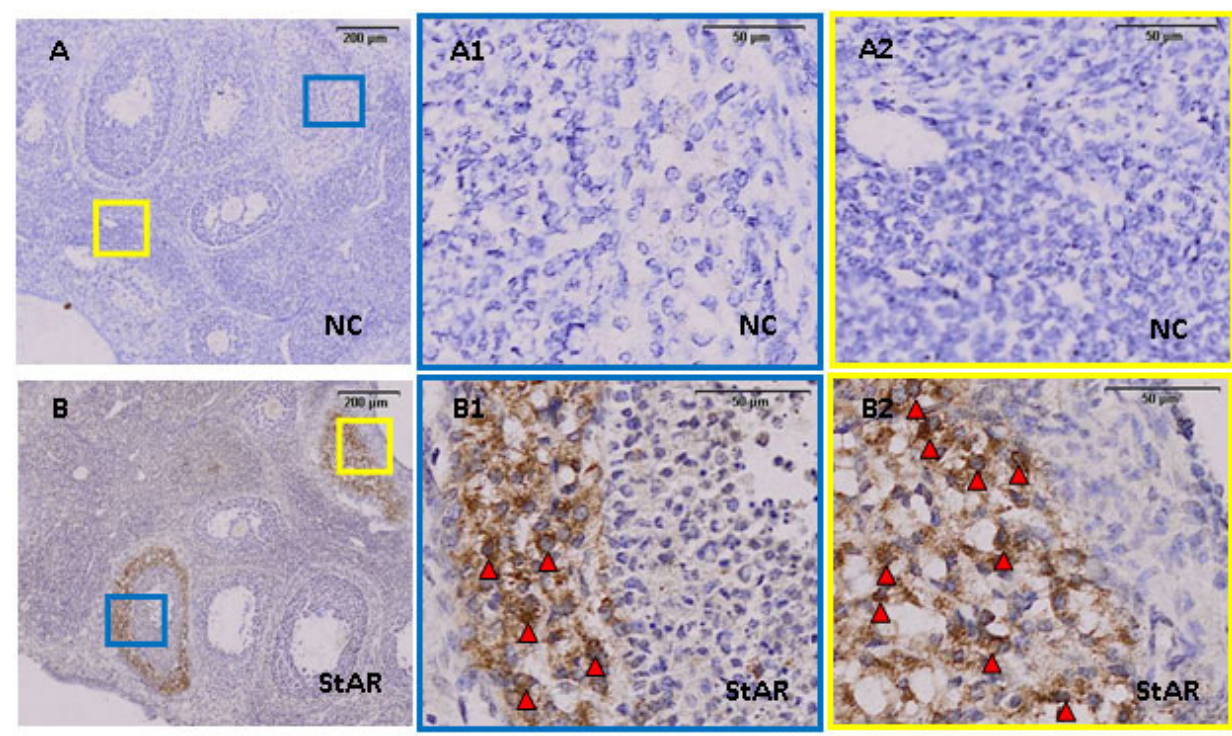

Figure 5. Immunolocalization of StAR in proestrus ovary. NC = negative control.
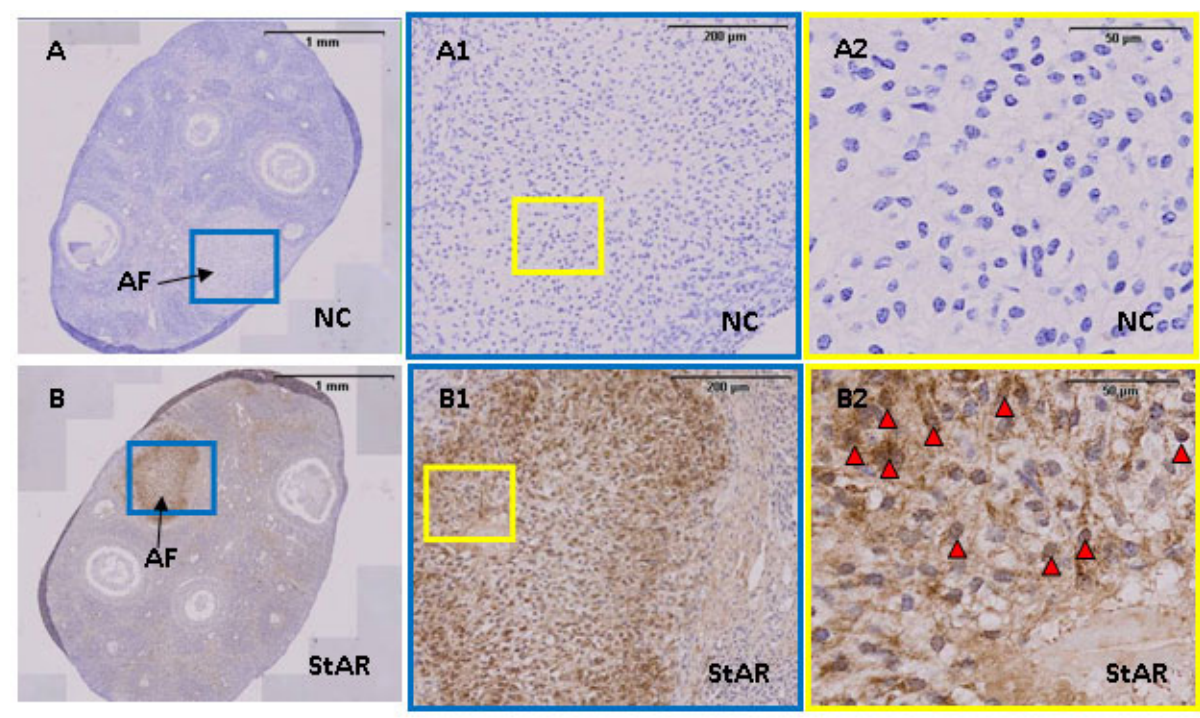

Figure 6. Immunolocalization of StAR in metestrus ovary. $\mathrm{NC}=$ negative control; $\mathrm{AF}=$ atretic follicle.

\section{Ovary MMP-2 and MMP-9 expression levels at proestrus and metestrus}

As shown in Figure 7, MMP-2 and MMP-9 were expressed in the proestrus ovaries. Compared with the negative control (Figure 7A, A1, A2, and A3), MMP-2 was expressed more in the proestrus ovaries than MMP-9 (Figure 7B, 7C). In the non-antral follicles, MMP-2 was expressed 
in the granulosa cells (Figure 7B1), while MMP-9 was expressed in the thecal cells (Figure 7C1). In the small antral primary follicles, MMP-2 was expressed in both granulosa cells and thecal cells (Figure 7B2), while MMP-9 was not expressed (Figure 7C2). In the atretic antral follicles, MMP-2 and MMP-9 were both expressed in the granulosa cells and basal membranes (Figure 7B3, C3).
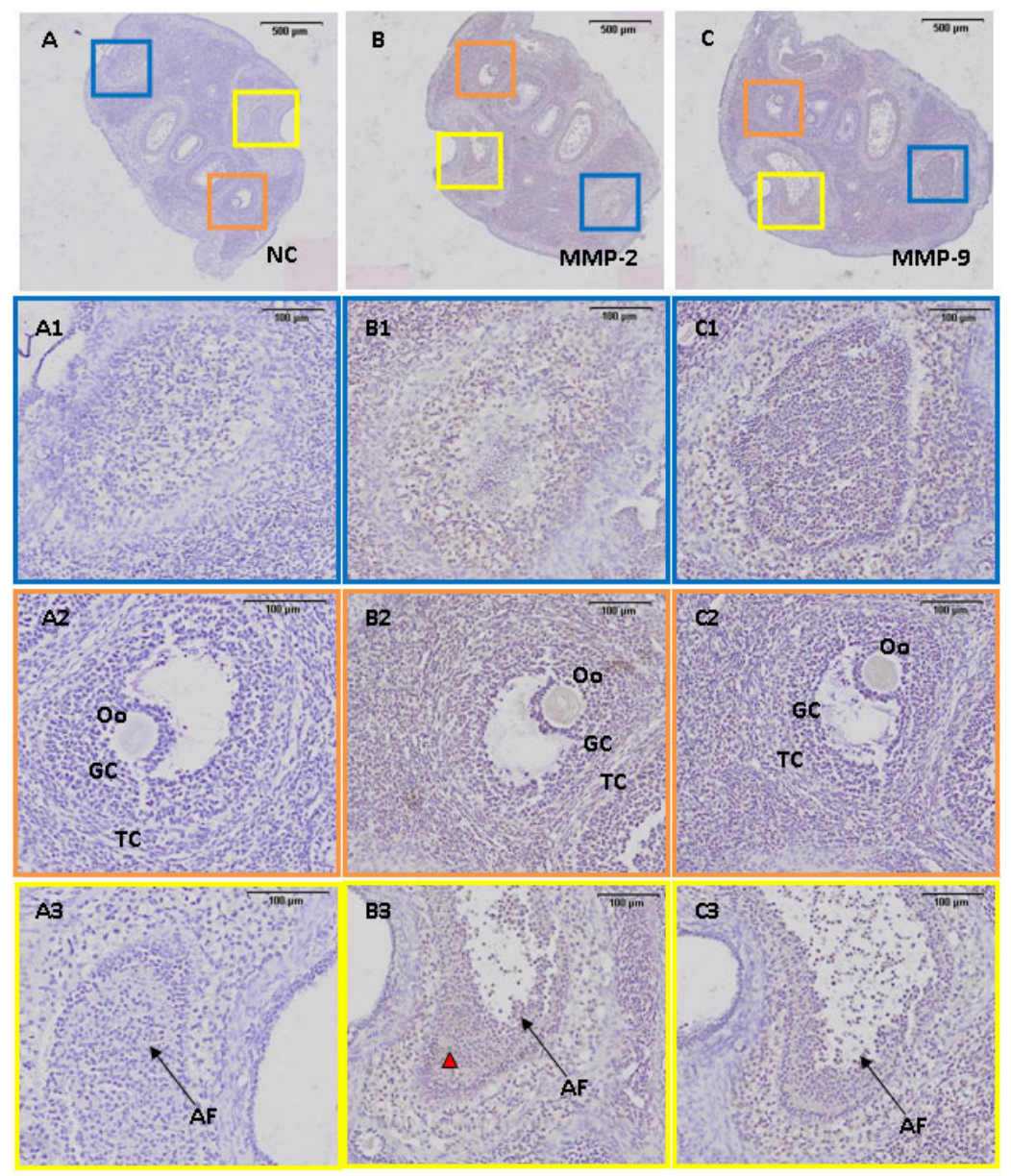

Figure 7. Immunolocalization of MMP-2 and MMP-9 in proestrus ovary. NC = negative control; Oo = oocyte; GC = granulosa cells; $\mathrm{TC}=$ theca cells; $\mathrm{AF}=$ atretic follicle.

Compared with the control group (Figure 8A, A1, A2, and A3), MMP-2 and MMP-9 were expressed in the metestrus ovaries (Figure 8B, C), and markedly in the ovary corpus luteum. In the late-atresia follicles (only transparent zones with irregular shapes), MMP-2 was weakly expressed in the basal membrane cell layer (Figure 8B1), while MMP-9 was not expressed (Figure 8C1). The expression levels of MMP-2 and MMP-9 in the small antral follicles were similar to those in the proestrus ovaries (Figure 8B2, C2). In the large antral follicles, MMP-2 and MMP-9 were both expressed in the separated follicular granulosa cells (Figure 8B3, C3). 

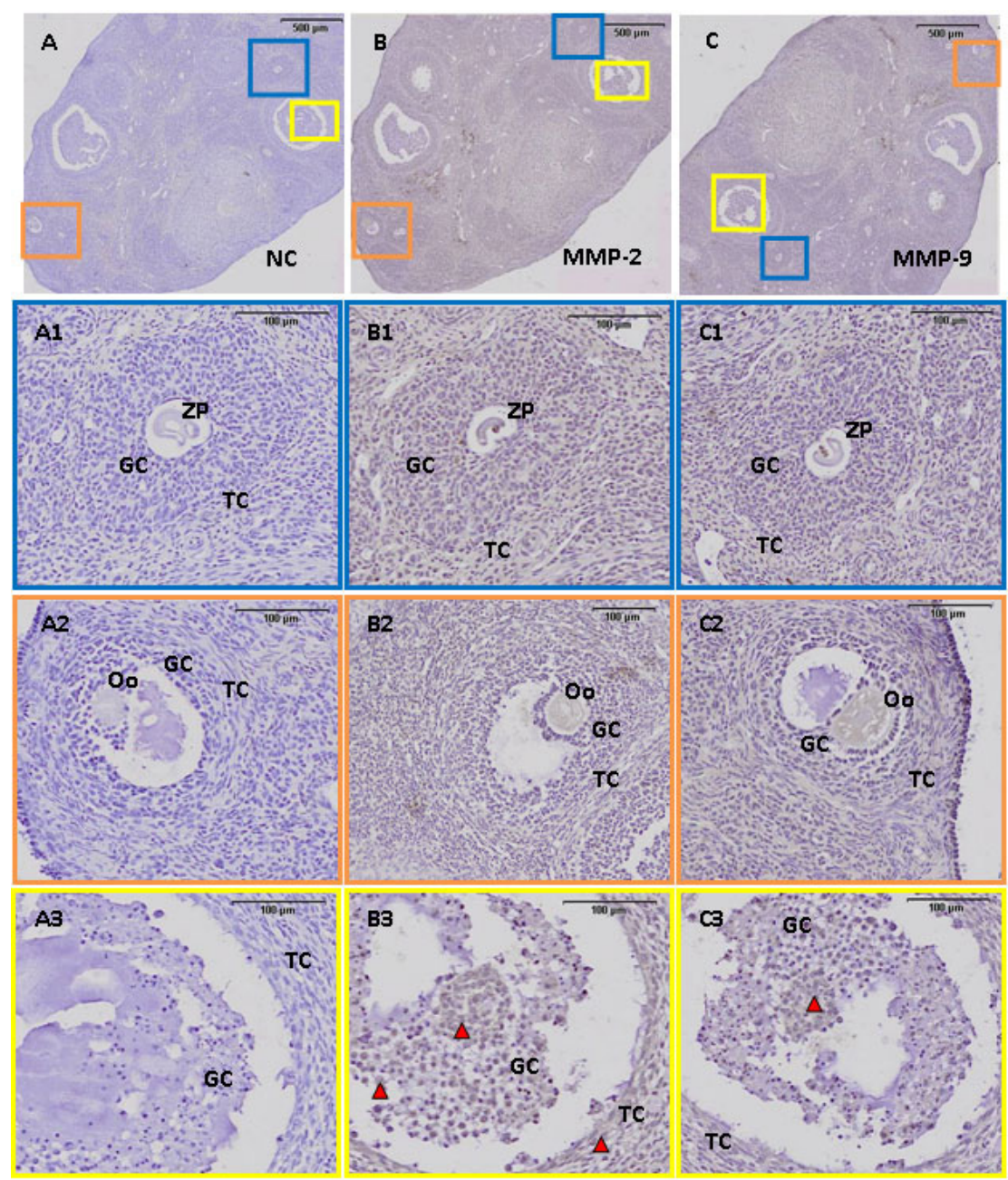

Figure 8. Immunolocalization of MMP-2 and MMP-9 in metestrus ovary. NC = negative control; Oo = oocyte; GC = granulosa cells; $\mathrm{TC}=$ theca cells; $\mathrm{AF}=$ atretic follicle; $\mathrm{ZP}=$ zona pellucida.

\section{DISCUSSION}

A normal estrous cycle is divided into four stages: proestrus, estrous, metestrus, and diestrus (Wang et al., 2010). The four stages are obviously affected by estrogen periodicity (Bland, 1980). In this study, the estrous cycle was easily determined according to vaginal orifice appearance and vaginal exfoliated cell smears. The results showed that the vaginal orifices at estrous were evidently opened, but were fully closed at an estrus. The vaginal orifices at estrous were red and swollen, but recovered and became white at an estrus. The vaginal exfoliated cells at estrous were fully-cornified ECs, but were WBCs at diestrus.

In the ovaries of adult guinea pigs with normal estrous cycles, the follicles mature gradually and in stages. Thus, follicles at different degrees of development are found in both proestrus and 
metestrus ovaries. The morphological characteristics are most apparent in metestrus ovaries, which contain the fresh corpus luteum formed after ovulation in mature follicles. The granulosa cell layer and the thecal cell layer in the large antral follicles are obviously separated. The proestrus ovaries ovulate normally as mature follicles. The ovaries show both normal mature antral follicles and large antral atretic follicles. The atretic follicles occur mostly at different atretic stages. The separation between the atretic follicle granulosa cell layer and the follicular membranes may be associated with the large-range fluctuation of sex hormones before ovulation (Shi et al., 1999).

The ovary steroids in female ovaries (e.g., testosterone, progesterone, and estradiol) are mainly synthesized in the steroidogenic acute regulatory pathway, which is regulated mostly by the StAR protein (Manna and Stocco, 2005). Thus, StAR is largely expressed in the large atretic follicles and corpus luteum; it is strongly expressed in the basal membrane cell layer of large atretic follicles, and the luteal cells in the corpus luteum, but not in the granulosa cell layers. These results indicate that at early follicular atresia and at the luteum stage, there is extensive synthesis and regulation of steroids.

The expression levels of MMP-2 and MMP-9 in the granulosa cells and basal membrane cells of pigs are very similar (Basiniet al., 2011). MMP-2 and MMP-9 are markedly expressed in the granulosa cell layer and the basal membrane cell layer in the antral follicles and atretic antral follicles of guinea pigs. Only weak MMP-2 expression and no evident MMP-9 expression was found in the basal membrane cell layer of the non-antral follicles at the late atretic stage. The expression of StAR in the ovary was also modestly correlated with MMP-2 and MMP-9 expression levels. At the early follicular atresia stage (or the atretic antral follicles stage) and in the corpus luteum, StAR, MMP-2, and MMP-9 were also evidently expressed. These results are consistent with the ovary observations in sheep and humans, and follicular atresia is accompanied by high intrafollicular MMP-2 and MMP-9 levels (Huet et al., 1998; Ben-Shlomo et al., 2003). The formation of the follicular corpus luteum necessitates abundant cell formation and angiogenesis. Thus, MMPs are involved in the formation of the follicular corpus luteum after ovulation in guinea pigs (Schmidt et al., 1995). The degeneration of the corpus luteum enhances the activity of MMPs (Boujradet al., 1995). However, high StAR expression in large antral follicles is not accompanied by MMP-2 or MMP-9 expression. Therefore, the specific correlation between ovary StAR and MMP-2 or MMP-9 expression in the ovaries of guinea pigs requires further study.

MMP-2 and MMP-9 are markedly expressed in the atretic antral follicles of guinea pig ovaries at proestrus, and are highly expressed in the ovary corpus luteum at metestrus.

\section{Conflicts of interest}

The authors declare no conflict of interest.

\section{ACKNOWLEDGMENTS}

Research supported in part by the Science and Technology Project of Zhejiang (\#2013C32053).

\section{REFERENCES}

Bagavandoss P (1998). Differential distribution of gelatinases and tissue inhibitor of metalloproteinase-1 in the rat ovary. J. Endocrinol. 158: 221-228. 
Baker AH, Zaltsman AB, George SJ and Newby AC (1998). Divergent effects of tissue inhibitor of metalloproteinase-1, -2 , or -3 overexpression on rat vascular smooth muscle cell invasion, proliferation, and death in vitro. TIMP-3 promotes apoptosis. J. Clin. Invest. 101: 1478-1487.

Basini G, Bussolati S, Baioni L and Grasselli F (2011). Gelatinases (MMP2 and MMP9) in swine antral follicle. Biofactors 37: 117-120.

Ben-Shlomo I, Goldman S and Shalev E (2003). Regulation of matrix metalloproteinase-9 (MMP-9), tissue inhibitor of MMP and progesterone secretion in luteinized granulosa cells from normally ovulating women with polycystic ovary disease. Fertil. Steril. 79: 694-701.

Bland KP (1980). Biphasic follicular growth in the guinea-pig oestrous cycle. J. Reprod. Fertil. 60: 73-76.

Bode W, Fernandez-Catalan C, Tschesche H, Grams F, et al. (1999). Structural properties of matrix metalloproteinases. Cell. Mol. Life Sci. 55: 639-652.

Boujrad N, Ogwuegbu SO, Garnier M, Lee CH, et al. (1995). Identification of a stimulator of steroid hormone synthesis isolated from testis. Science 268: 1609-1612.

Brew K, Dinakarpandian D and Nagase H (2000). Tissue inhibitors of metalloproteinases: evolution, structure and function. Biochim. Biophys. Acta 1477: 267-283.

Clark BJ, Combs R, Hales KH, Hales DB, et al. (1997). Inhibition of transcription affects synthesis of steroidogenic acute regulatory protein and steroidogenesis in MA-10 mouse Leydig tumor cells. Endocrinology 138: 4893-4901.

Cooke RG 3rd, Nothnick WB, Komar C, Burns P, et al. (1999). Collagenase and gelatinase messenger ribonucleic acid expression and activity during follicular development in the rat ovary. Biol. Reprod. 61: 1309-1316.

Di Nezza LA, Misajon A, Zhang J, Jobling T, et al. (2002). Presence of active gelatinases in endometrial carcinoma and correlation of matrix metalloproteinase expression with increasing tumor grade and invasion. Cancer 94: $1466-1475$.

García R, Ballesteros LM, Hernández-Pérez O, Rosales AM, et al. (1997). Metalloproteinase activity during growth, maturation and atresia in the ovarian follicles of the goat. Anim. Reprod. Sci. 47: 211-228.

Huet C, Monget P, Pisselet C, Hennequet C, et al. (1998). Chronology of events accompanying follicular atresia in hypophysectomized ewes. Changes in levels of steroidogenic enzymes, connexin 43, insulin-like growth factor II/mannose 6 phosphate receptor, extracellular matrix components, and matrix metalloproteinases. Biol. Reprod. 58: $175-185$.

Jones GT (2014). Matrix metalloproteinases in biologic samples. Adv. Clin. Chem. 65: 199-219.

Kessenbrock K, Plaks V and Werb Z (2010). Matrix metalloproteinases: regulators of the tumor microenvironment. Cell 141: 52-67.

Kulduk E, Eren E, Soy F, Dundar R, et al. (2014). Histological analysis of the effects of anti-adhesive haemostatic agents on the middle ear of the guinea pig. J. Laryngol. Otol. 128: 885-891.

Lohi J, Wilson CL, Roby JD and Parks WC (2001). Epilysin, a novel human matrix metalloproteinase (MMP-28) expressed in testis and keratinocytes and in response to injury. J. Biol. Chem. 276: 10134-10144.

Manna PR and Stocco DM (2005). Regulation of the steroidogenic acute regulatory protein expression: functional and physiological consequences. Curr. Drug Targets Immune Endocr. Metabol. Disord. 5: 93-108.

Massova I, Kotra LP, Fridman R and Mobashery S (1998). Matrix metalloproteinases: structures, evolution, and diversification. FASEB J. 12: 1075-1095.

McCaffery FH, Leask R, Riley SC and Telfer EE (2000). Culture of bovine preantral follicles in a serum-free system: markers for assessment of growth and development. Biol. Reprod. 63: 267-273.

Nagase H and Woessner JF Jr (1999). Matrix metalloproteinases. J. Biol. Chem. 274: 21491-21494.

Nagase H, Visse R and Murphy G (2006). Structure and function of matrix metalloproteinases and TIMPs. Cardiovasc. Res. 69: 562-573.

Raza SL and Cornelius LA (2000). Matrix metalloproteinases: pro- and anti-angiogenic activities. J. Invest. Dermat. Symp. Proceed. 5: 47-54.

Rivera S, Khrestchatisky M, Kaczmarek L, Rosenberg GA, et al. (2010). Metzincin proteases and their inhibitors: foes or friends in nervous system physiology? J. Neurosci. 30: 15337-15357.

Roy R, Zhang B and Moses MA (2006). Making the cut: protease-mediated regulation of angiogenesis. Exp. Cell. Res. 312: 608-622.

Roy R, Yang J and Moses MA (2009). Matrix metalloproteinases as novel biomarkers and potential therapeutic targets in human cancer. J. Clin. Oncol. 27: 5287-5297.

Schmidt L, Münster K and Helm P (1995). Infertility and the seeking of infertility treatment in a representative population.Br. J. Obstet. Gynaecol. 102: 978-984.

Shalev E, Goldman S and Ben-Shlomo I (2001). The balance between MMP-9 and MMP-2 and their tissue inhibitor (TIMP)-1 in luteinized granulosa cells: comparison between women with PCOS and normal ovulatory women. Mol. Hum. Reprod. 7: $325-331$ 
Shi F, Ozawa M, Komura H, Yang P, et al. (1999). Secretion of ovarian inhibin and its physiologic roles in the regulation of follicle-stimulating hormone secretion during the estrous cycle of the female guinea pig. Biol. Reprod. 60: 78-84.

Shi YB, Li Q, Damjanovski S, Amano T, et al. (1998). Regulation of apoptosis during development: input from the extracellular matrix (review). Int. J. Mol. Med. 2: 273-282.

Sternlicht MD, Bissell MJ and Werb Z (2000). The matrix metalloproteinase stromelysin-1 acts as a natural mammary tumor promoter. Oncogene 19: 1102-1113.

Stetler-Stevenson WG (1994). Proteases and their inhibitors in invasion and metastasis Progelatinase A activation during tumor cell invasion. Invas. Metast. 14: 259-268.

Sun SY, Zhang W, Han X, Huang RH, et al. (2014). Cell proliferation and apoptosis in the fetal and neonatal ovary of guinea pigs. Genet. Mol. Res. 13: 1570-1578.

vanKan CM, de Vries JI, Lüchinger AB, Mulder EJ, et al. (2009). Ontogeny of fetal movements in the guinea pig. Physiol. Behav. 98: 338-344.

Vu TH and Werb Z (2000). Matrix metalloproteinases: effectors of development and normal physiology. Genes Dev. 14: 21232133.

Wang W, Liu HL, Tian W, Zhang FF, et al. (2010). Morphologic observation and classification criteria of atretic follicles in guinea pigs. J. Zhejiang Univ. Sci. B 11: 307-314. 